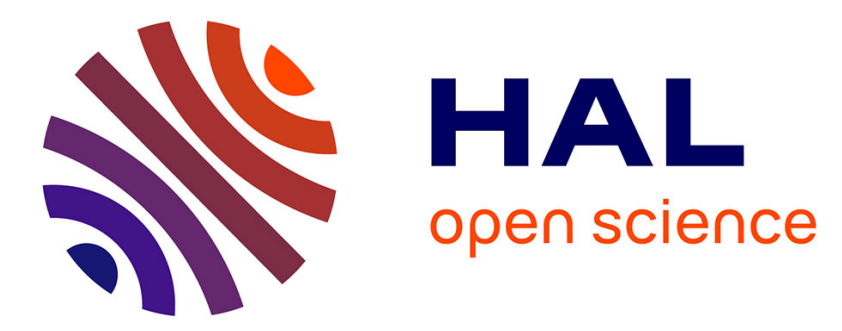

\title{
Deconstructing the chronicles : rumours and extreme violence during the siege of Meaux (1421-1422)
}

\author{
Boris Bove
}

\section{To cite this version:}

Boris Bove. Deconstructing the chronicles : rumours and extreme violence during the siege of Meaux (1421-1422). French History, 2010, 24 (4), pp.501-523. halshs-00638663v2

\section{HAL Id: halshs-00638663 \\ https://shs.hal.science/halshs-00638663v2}

Submitted on 11 Nov 2011

HAL is a multi-disciplinary open access archive for the deposit and dissemination of scientific research documents, whether they are published or not. The documents may come from teaching and research institutions in France or abroad, or from public or private research centers.
L'archive ouverte pluridisciplinaire HAL, est destinée au dépôt et à la diffusion de documents scientifiques de niveau recherche, publiés ou non, émanant des établissements d'enseignement et de recherche français ou étrangers, des laboratoires publics ou privés. 


\section{Deconstructing the chronicles : rumours and extreme violence around the siege of Meaux (1421-1422)}

RESUME : Le présent article se propose d'interroger la réalité de la crise de la fin du Moyen Âge à travers l'examen attentif des crimes terrifiants d'un capitaine armagnac lors du siège de Meaux en 1421-1422, rapportés par le Journal d'un bourgeois de Paris. Ces crimes s'inscrivent dans un discours largement répandu dans les chroniques, qui soulignent l'extrême violence de leur époque, et ont souvent été crues sur parole par les historiens. Le récit du Bourgeois de Paris est en fait un entrelacs serré de stéréotypes visant à discréditer l'adversaire ; la comparaison avec les nombreuses chroniques qui rapportent les mêmes faits prouve même que l'anecdote qu'il rapporte est un pur fantasme. Ce cas invite à penser que les derniers siècles du Moyen Âge furent traversés par des vagues de peur liées aux troubles répétés de l'ordre public, plus que le théâtre ordinaire d'une violence extrême.

La fin du Moyen Âge est généralement décrite comme une période de crise, et il n'y a pas lieu de remettre en question ce constat pour la France des XIVe et XVe siècles: la peste, la guerre de Cent Ans et l'impôt ont saigné la population et désorganisé l'économie. La question est plutôt de savoir jusqu'où s'étend la crise. Autrement dit, se limite-elle à certains secteurs ou faut-il considérer que l'on a affaire à une crise systémique qui est aussi celle de la civilisation médiévale toute entière, comme l'a parfois fait l'historiographie d'inspiration marxiste à partir des années $1970^{1}$. La tendance historiographique actuelle en France est plutôt à la nuance, mais il est encore des partisans d'une crise totale, comme en témoigne le dernier livre de Guy Bois : pour lui, la crise du féodalisme dans les années 1300 entrâne le blocage de l'économie agraire, la guerre, les épidémies, les famines, l'explosion de la pauvreté et de la criminalité, ainsi que l'effondrement du commerce et de l'industrie ; la peste suscite les «manifestations aberrantes » des flagellants et des pensées macabres, mais elle provoque aussi l'angoisse et le pessimisme, exploité par les clercs dans le cadre d'une pastorale de la peur, bref un « choc culturel » qui libère les pires pulsions des hommes, «sans quoi on ne comprendrait pas les déchaînements ultérieurs de la guerre civile et des écorcheurs $»^{2}$. Il est vrai que les descriptions crépusculaires d'une violence paroxystique ne manquent pas dans les chroniques médiévales françaises de la fin du Moyen Âge, mais faut-il les prendre au pied de la lettre lorsqu'elles évoquent les pillages, les sacrilèges, les incendies, les vols, les rapts, les viols, les tortures, voire d'anthropophagie qui semblent se développer à cette époque ?

On voudrait ici discuter la réalité de la violence extrême évoquée dans les chroniques par l'étude minutieuse d'une anecdote terrifiante rapportée par le Journal d'un bourgeois de Paris, souvent reprise par les historiens, presque toujours sans critique. En effet, le Journal a une place de choix parmi les sources auxquelles ils ont puisé leurs convictions alarmistes sur la fin du Moyen $\hat{A}_{g e}{ }^{3}$. L'historiographie de la fin $\mathrm{du} \mathrm{XX}^{\mathrm{e}}$ siècle, plus soucieuse des représentations que des faits, est en général plus méfiante. Colette Beaune a souligné la fragilité de l'information de cette chronique, tandis que l'usage récurrent, partial et allusif que fait son auteur des stéréotypes de la 
grande criminalité invite à douter de la réalité de ses affirmations ${ }^{4}$ - ainsi les Armagnacs sont-ils systématiquement accusés des pires crimes avec des formules récurrentes ${ }^{5}$. Mais toutes les accusations dont il accable le parti adverse ne sont pas stéréotypées et il demeure quelques récits atroces dont on ne sait que penser parce qu'ils relatent des anecdotes circonstanciées, précises et originales. Parmi eux, la description de la tyrannie du bâtard de Vaurus, un capitaine de routiers qui a terrorisé les habitants d'Îlede-France entre 1418 et $1422^{6}$.

L'auteur du Journal d'un bourgeois de Paris est anonyme. Il a été improprement qualifié de «bourgeois » par ses premiers éditeurs, mais il semble qu'il s'agisse plutôt d'un clerc parisien, proche de l'université et de la reine Isabeau. C'est un propriétaire très attentif aux événements parisiens et aux ravages de la guerre en Île-de-France, mais il manifeste aussi beaucoup de compassion pour les souffrances du menu peuple. Comme beaucoup de Parisiens, il est bourguignon de cœur, et farouchement hostile au parti armagnac. Ses sympathies politiques le conduisent à être plutôt favorable aux Anglais, dès lors qu'ils deviennent les alliés du duc de Bourgogne Philippe le Bon après l'assassinat de son père par les Armagnacs en 1419. La structure de son Journal est strictement chronologique. Il a été rédigé au jour le jour, mais certains passages ont été écrits après coup : ils correspondent en général à des morceaux de bravoure rhétorique qui contrastent avec la sécheresse des notes quotidiennes. Le récit de l'infamie du bâtard de Vaurus en est l'exemple même'.

L'anecdote s'inscrit dans la période troublée qui suit la conquête anglaise de la partie septentrionale du royaume de France, entre 1415 et 1422. La victoire d'Henri V est reconnue en 1420 par le traité de Troyes qui lui donne la main de la fille de Charles VI, ainsi que le gouvernement de la France ; il prévoit aussi que le petit-fils des deux rois, à naître de cette union, serait roi de France et d'Angleterre. C'était sans compter sans la résistance du dauphin Charles, réfugié dans le «royaume de Bourges » au sud de la Loire et soutenu par le parti armagnac : non seulement le jeune Charles dénonce le traité et se considère toujours comme l'héritier légitime de la couronne de France, mais ses partisans résistent avec la dernière énergie à l'avancée anglaise. Le traité de Troyes n'a pas en effet provoqué l'arrêt des combats, puisqu'il était dénoncé par les Armagnacs. En Île-de-France, les Bourguignon ont ouvert les portes de la capitale aux Anglais, mais bon nombre de place fortes alentours étaient toujours aux mains de capitaines fidèles au Dauphin qui menaient une guerre d'usure aux anglobourguignons dans les campagnes. Parmi eux, le bâtard de Vaurus, capitaine de la forteresse de Meaux sur la Marne. Henri V vint mettre le siège devant la ville en septembre 1421 et finit par l'emporter au bout de neuf mois. Il fit alors décapiter le bâtard de Vaurus et suspendre son corps, drapé dans son étendard, à un orme au dehors de la ville, tandis que sa tête était fichée sur sa lance à la cime d'un arbre.

Cette exécution exemplaire se justifie, selon l'auteur du Journal d'un bourgeois de Paris, par la cruauté du supplicié, qui avait coutume de rançonner les paysans alentours et de les pendre à son orme lorsqu'ils ne pouvaient payer. Il en veut pour preuve une anecdote qu'il développe ensuite. Un jour, le bâtard de Vaurus, qui «était plus cruel tyran qu'oncques guère fut Néron ou autre », captura un jeune homme qui labourait son champ et le fit torturer à Meaux jusqu'à ce qu'il accepte de payer une rançon trois fois supérieure à sa fortune. Sa femme, jeune épousée qui attendait un enfant, tenta en vain d'attendrir le cœur du tyran, et dut se résoudre à réunir la somme en question après avoir quitté en pleurant l'homme qu'elle aimait. Elle fit aussi vite que 
son état et l'énormité de la rançon le lui permettaient, si bien qu'elle apporta la somme au bâtard de Vaurus huit jours après l'expiration du délai, en mars 1421 (n. st). Celui-ci avait fait entre-temps exécuter le laboureur au jour dit, mais n'en informa la jeune femme qu'après qu'elle eut payé la rançon. À l'annonce de la mort de son époux, elle devint folle de douleur et insulta son bourreau. Celui-ci la fit alors battre et amener immédiatement à l'orme, où il l'attacha au tronc et lui fit couper ses vêtement jusqu'au nombril, dénudant ainsi son sexe et son ventre. Au-dessus d'elle, quatre-vingt ou cent pendus oscillaient au vent et heurtaient parfois sa tête, lui faisant pousser des hurlements de terreur qu'on entendait depuis la ville. La nuit tomba. Elle cria longtemps, mais personne n'osait intervenir, par crainte du bâtard de Vaurus. «En ces douloureux cris le mal de son enfant la prit, tant pour la douleur de ses cris, comme pour la froidure du vent qui par-dessous l'assaillait de toute part, ces ondées la hâtèrent plus et plus ; si cria tant haut que les loups, qui là repairaient pour la charognes, vinrent à son cri droit à elle, et de toute part l'assaillirent, especialement au pauvre ventre qui découvert était, et lui ouvrirent à leurs cruelles dents, et tirèrent l'enfant dehors par pièces, et le remenant de son corps dépecèrent tout. Ainsi fina cette pauvre créature et autres assez, et ce fut au mois de mars en Carême, l'an $1420 »^{8}$.

L'épisode est connu, mais n'a jamais vraiment été discuté. Les livres d'histoire d'Ancien Régime, attachés à donner une version compatible avec l'honneur du roi, sont assez pudiques sur l'événement : ils se contentent de mentionner, sans détailler, que cet Armagnac était un soldat brave, mais cruel, qui a été exécuté pour ses méfaits ${ }^{9}$. Le récit du Journal d'un bourgeois de Paris eut cependant une grande fortune dans l'historiographie romantique qui y trouva l'illustration parfaite d'un Moyen Âge crépusculaire. Sismondi et Barante popularisèrent 1'histoire ${ }^{10}$, qui paraît avoir connu une certaine célébrité dans les années 1830, puisqu'elle est l'objet d'une dissertation écrite par un élève de seize ans en classe de rhétorique ${ }^{11}$. Sismondi et Barante prennent l'histoire au pied de lettre et l'authentifient par la même occasion, ce qui permet à leurs successeurs d'affirmer « [qu'] un pareil trait de férocité est si révoltant qu'on hésiterait à en croire Vaurus même capable s'il n'était mentionné par M. de Barante dans sa belle histoire des ducs de Bourgogne ${ }^{12}$. Michelet n'échappe pas à la règle et Vaurus entre dans la culture littéraire des écrivains du XIX ${ }^{\mathrm{e}}$ siècle comme une référence de cruauté ${ }^{13}$. Depuis, l'épisode du bâtard de Vaurus continue à être pris au pied de la lettre ${ }^{14}$. Seule Colette Beaune, se fondant sur une intuition plus que sur une démonstration, affirme qu'il relève du «fantasme ${ }^{15}$. Faut-il aussi ranger ce récit dans le dossier des fabulations de chroniqueurs terrifiés par la crise de l'ordre public ? Ce problème soulève deux questions : celle de la réalité du fait, mais aussi celle de la puissance d'évocation du récit qui semble avoir fasciné la plupart des historiens, au point d'engourdir leur sens critique.

Vaurus a bien existé. C'est un personnage très secondaire de la guerre de Cent Ans, mais on possède tout de même trois pièces comptables relatives à son service militaire. Le 30 novembre 1417, le Dauphin Charles donne à son «bien amé le bastart de Warus, escuier », la somme de 250 1.t., pour ses bons et agréables services faits en temps de guerre, comme pour l'aider à entretenir les gens d'armes et de trait de sa compagnie $^{16}$. Deux autres documents prouvent qu'il est entre le 26 août et le 30 novembre 1419, avec 19 écuyers de sa chambre, dans la compagnie d'Arnaud de Barbazan, alors capitaine de Melun ${ }^{17}$. Ce dernier, gascon originaire de Bigorre, conseiller et chambellan du Dauphin, était mêlé de près à l'assassinat de Jean sans Peur, 
puisque Philippe le Bon réclama ensuite officiellement justice contre lui à Henri $\mathrm{V}^{18}$. En 1420, Barbazan était lieutenant du Dauphin dans les bailliages de Meaux, Melun et Senlis ; il tint la ville de Melun jusqu'à sa reddition honorable en octobre $1420^{19}$. En mars 1421, Barbazan est prisonnier des Anglais, mais Vaurus a probablement été nommé capitaine de Meaux sur son ordre. Le qualificatif de «bâtard » et le titre d'écuyer de Vaurus révèlent qu'il est d'ascendance noble. Pierre de Fénin dit même que sa renommée était plus grande que celle de son frère Denis, "qui estoit grand seigneur $»^{20}$. Le jugement est un peu exagéré, car la famille est inconnue des généalogistes ${ }^{21}$ et absente des archives centrales de la monarchie ${ }^{22}$ : peut-être est-elle de petite noblesse ? Jean Juvénal des Ursins affirme que c'est un gascon au service de feu Bernard VII d'Armagnac ${ }^{23}$, ce que corroborerait sa présence dans la compagnie d'Arnaud de Barbazan. Sa proximité avec ce dernier en fait un armagnac de la plus belle eau. Il est possible que le nom Vaurus soit une francisation de Lavaur (Vaurum), cheflieu d'un diocèse dans le Tarn, sans que cette piste ait été plus fructueuse. On est donc surtout renseigné sur le bâtard de Vaurus par la trentaine de chroniques qui racontent le siège de Meaux, car la chute de la ville a marqué un tournant dans l'offensive anglaise en Île-de-France.

L'existence historique de Vaurus et du siège de Meaux ne signifient pas pour autant l'exactitude des propos du Journal. L'examen du texte lui-même, comme de son contexte, permet au contraire d'en douter sérieusement.

Il faut lire le texte original du Bourgeois de Paris pour sentir la dimension archétypale de son récit, qui doit beaucoup à sa qualité littéraire. Tout en prenant le récit au pied de la lettre, Michelet l'avait bien identifiée, lui qui parlait de Vaurus comme d'une «espèce d'ogre », d'une «férocité fabuleuse ${ }^{24}$. On ne peut qu'être frappé en effet par la perfection rhétorique de l'histoire. Elle oppose, en un contraste parfait, un monde humain, civilisé, chrétien, vertueux, fondé sur l'amour, visant à la création, à un monde animal, sauvage, pervers, fondé sur la haine, visant à la destruction. Cette opposition se dessine tant au niveau du statut des personnages que de leurs fonctions et de leurs actions. La victime exerce une activité pacifique et productive ; le bourreau est un homme de guerre pillard. La jeune femme est enceinte et sa fertilité est l'avenir de la chrétienté; le capitaine de Meaux au contraire apporte la mort et les troubles qu'occasionnent les gens d'armes semblent vouloir détruire la société. Les jeunes gens forment un couple légitime qui est la cellule fondatrice de la société ; Vaurus est un bâtard. Ils sont d'ascendance non noble mais vertueux; le capitaine est d'ascendance noble mais pervers. Les époux sont aimants ; il est sans pitié. Elle est innocente ; il est coupable. Elle meurt; il vit. Les victimes cumulent tous les éléments de la bonne renommée (respect du sacrement du mariage, honnêteté, aisance, travail, conformité aux normes du groupe), au contraire de leur bourreau qui est son seulement un bâtard, mais encore un homme d'armes, donc doublement rejeté au marges de la société par l'opinion ${ }^{25}$.

Le portrait de Vaurus que dessine le Bourgeois à l'occasion de ce récit est aussi une épure idéologique : non seulement le capitaine de Meaux incarne l'essence du tyran, mais c'est aussi un monstre inhumain. L'accusation de tyrannie n'est guère surprenante, puisque c'est ainsi que le Bourgeois considère tous les hommes d'armes qui vivent sur le pays ${ }^{26}$. La définition de la tyrannie selon l'auteur du Journal tient surtout dans l'abus de pouvoir et de la force militaire, ce qui se traduit par une litanie en général stéréotypée dénonçant les meurtres, les incendies, les viols et les sacrilèges des routiers qui mettent 
en péril la société en bafouant ses règles les plus sacrées. Une fois n'est pas coutume, l'auteur du Journal prouve son accusation par le récit circonstancié des méfaits du capitaine armagnac. Le souci de justification serait crédible s'il ne dressait le portrait d'un tyran parfait. L'action politique et militaire de Vaurus est en effet illégitime à tous les niveaux. D'abord, il laisse libre cours à son ubris de capitaine de Meaux dans une parodie de justice: il s'arroge le droit de juger, alors que celui-ci appartient normalement au bailli local; il s'adjoint le service d'un bourreau, mais "s'il ne trouvait bourrel prêt, lui-même les pendait »; il fait pendre à un orme, qui est un arbre de justice $^{27}$, non un gibet; il pend enfin ses victimes sans les laisser se préparer chrétiennement à la mort. Ensuite, il transgresse les tabous fondateurs de la société : aucune société ne se donne le droit d'attenter à une femme enceinte, non seulement parce que son enfant est innocent, mais aussi parce qu'il est le gage de la perpétuation du groupe ${ }^{28}$. À ce premier tabou s'ajoute celui du Carême. Le crime du bâtard de Vaurus est d'autant plus condamnable dans ce contexte de purification religieuse qu'il a une dimension sexuelle évidente, même s'il ne s'agit pas d'un viol - mais dénuder sa victime jusqu'au nombril n'a aucune justification judiciaire. On pourrait ajouter enfin, au regard de la culture politique de l'époque sur la tyrannie fondée sur Aristote, que la bâtardise de Vaurus en fait un tyran à la fois illégitime dans son pouvoir et dans son action. Il incarne donc l'essence du tyran et rejoint en cela Néron auquel le Journal le compare, après Bernard d'Armagnac et les grands seigneurs responsables de la guerre civile. Plus qu'une comparaison, le récit établit une analogie par le crime entre le capitaine de Meaux et Néron qui, selon Jacques de Voragine dans son récit de la vie de saint Pierre, « ordonna de tuer sa mère et de l'éventrer, pour voir de quelle manière elle l'avait tenu au chaud dans sa matrice $»^{29}$.

Vaurus est un tyran, mais le récit du Bourgeois le range en outre du côté du monde sauvage et infernal, puisque ses auxiliaires de justice sont des loups. Il y a naturellement des loups dans les campagnes franciliennes à cette époque ${ }^{30}$. Toutefois les mentions de loups dans le Journal dépassent la simple observation éthologique, car l'auteur mêle à ces observations réelles des croyances si bien enracinées dans la culture qu'elles infléchissent son jugement. Il est très improbable par exemple que les loups s'attaquent aux hommes adultes et aux bergers plutôt qu'aux troupeaux comme l'affirme pourtant le Journal - cela reflète plutôt la certitude bien ancrée au Moyen Âge que le loup est anthropophage ${ }^{31}$, si bien qu'il est volontiers assimilé à l'ogre dévoreur d'enfants ${ }^{32}$. Les loups ont donc un étrange comportement dans le Journal, parce qu'ils sont les agents du diable à une époque où l'auteur pressent une fin du monde imminente, entre peste et guerre. Si l'on se laisse porter par la logique du récit, on arrive vite à la conclusion que Vaurus commande aux loups, parce qu'il est lui-même un loup ! Le capitaine de Meaux n'est-il pas un bâtard, comme le loup ${ }^{33}$ ? N'a-t-il pas comme lui a un appétit insatiable ? D'ailleurs la «lycanthropisation » du bâtard de Vaurus renvoie, dans le contexte de la guerre civile, au parti de Louis d'Orléans qui affichait entre 1389 et 1399 une devise au loup que la propagande bourguignonne avait retourné contre ses fidèles depuis 1407 au moins ${ }^{34}$. Dans l'imaginaire occidental, le loup se singularise par son caractère dévorateur, ce qui permettait aux Bourguignons de dénoncer de manière imagée la rapacité fiscale du duc d'Orléans ${ }^{35}$. Dans la culture politique les partisans du duc de Bourgogne, comme l'est l'auteur du Journal, les Armagnac sont donc des loups - que le bâtard de Vaurus leur délègue l'exécution de ses victimes semble donc très cohérent. D'une manière générale, le Bourgeois qualifie volontiers les tyrans de loups, comme beaucoup de ses contemporains ${ }^{36}$ : ainsi Philippe 
de Morvilliers, premier président du Parlement responsable du gouvernement de Paris en 1421, ou les brigands plus ou moins soldés qui pillent les campagnes en 1432.

L'image du tyran se superpose donc à celle du lycanthrope pour en faire un ennemi du genre humain qu'il paraît vouloir faire disparaître. À cet égard, la scène de dévoration par les loups de l'enfant en train de naître peut se lire comme un accouchement contre-nature qui va contre les lois divines et menace d'ordre du monde : l'homme à naître passe du ventre de sa mère au ventre du loup, via la bouche d'Enfer. Il faut peut-être y voir un écho du passage de l'Apocalypse (12:5) où «en arrêt devant la Femme en travail, le Dragon s'apprête à dévorer son enfant aussitôt né ». Vaurus et ses loups apparaissent donc comme des auxiliaires objectifs de l'antique Ennemi, dans une lutte apocalyptique déclarée au Bien par les forces du Mal. Le capitaine de Meaux n'est pas explicitement identifié à l'Antéchrist, parce que ses actions parlent pour lui, mais le Journal associe à deux reprises Bernard d'Armagnac au Diable et lorsque le Bourgeois est plus allusif sur les méfaits des hommes de guerre, ceux-ci sont comparés aux Sarrasins, aux païens ou aux membres d'Antéchrist ${ }^{37}$.

Le récit du Bourgeois de Paris est donc très fortement informé de stéréotypes culturels et politiques qui font douter de la véracité des faits. Ceux-ci sont trop conformes à l'idée que se faisaient les Parisiens des routiers armagnacs de Meaux pour ne pas être suspects. Les doutes que fait naître la critique interne du document sont confirmés par l'étude des autres témoignages relatifs au siège de Meaux, qui permettent d'établir la dimension fantasmatique de l'histoire de la femme dévorée par les loups.

Le siège de Meaux est relaté dans 27 chroniques au moins, et Vaurus apparaît dans 15 d'entre elles. Leur témoignage est à peu près concordant, à quelques détails près qui permettent de les répartir en quatre groupes: les chroniques anglaises, bourguignonnes, armagnacs et parisiennes (que les chroniqueurs soient favorables aux anglo-bourguignons, ou légitimistes) donnent chacune une interprétation différente de l'événement (annexe 2).

Les chroniques anglaises forment le groupe le moins cohérent et sont décevantes pour notre propos. Les Chroniques de Londres sont tardives et se contentent le plus souvent de mentionner la prise de Meaux, à l'exception de la Grande chronique de Londres qui a connaissance du traité de reddition de la garnison de Meaux, archivé en Angleterre. C'est dans les Vies d'Henri $V$ qu'il faut chercher l'information détaillée sur le déroulement du siège : celle de l'humaniste italien Tito Livio da Forli et surtout celle du pseudo Thomas d'Elmham qui est beaucoup plus exacte et prolixe pour les années 1420-1422. Toutes deux ont été écrites peu après le règne d'Henri $\mathrm{V}$ et puisent à des sources de première main, en particulier le pseudo Thomas d'Elmham qui tire probablement son récit du siège de Meaux d'un des protagonistes, Walter de Hungerford $^{38}$. Autant cette source est prolixe sur les exploits guerriers d'Henri V, comme on pouvait s'y attendre, autant elle est avare de détails sur les participants au siège de Meaux - elle n'est pas non plus exempte d'erreurs, puisqu'elle affirme que Denis de Vaurus a été pendu au même arbre que son parent, alors qu'il a été exécuté plus tard à Paris. Vaurus y est décrit comme un tyran cruel qui ravageait la contrée et pendait des innocents qui refusaient de payer rançon, mais de donne pas de détails et présente les faits de façon très partisane.

Les récits les plus complets, les plus cohérents et les moins polémiques sont sans aucun doute ceux des chroniqueurs bourguignons, et en particulier celui de Jean de Wavrin (1400-1475), contemporain des événements qui a servi dans les armées de 
Philippe le Bon et du roi d'Angleterre entre 1428 et 1435 . On peut juger de leur sérieux au fait qu'ils sont les seuls, avec la Grande chronique de Londres, à donner les détails du traité de reddition, et la collation avec le texte original conservé dans les archives anglaise montre une concordance quasi-parfaite (annexe 3$)^{39}$. Les Bourguignons sont bien informés parce que nombre d'entre eux participent au siège, comme Jean de Luxembourg qui était venu traiter de la délivrance de son frère, prisonnier d'un des capitaines retranchés dans Meaux. Mais d'autres capitaines bourguignons y étaient aussi, comme le sire de Châtillon ou le bâtard de Thian. Leur présence s'explique par l'alliance de raison de Philippe le Bon avec Henri V après le meurtre de Montereau : l'armée bourguignonne combat les Dauphinois aux côté des Anglais depuis le traité de Troyes - c'est pourquoi le traité de reddition de la garnison de Meaux stipule que les responsables de la mort de Jean sans Peur, s'il s'en trouve parmi les vaincus, seront livrés à Henri V. Toutefois le duc de Bourgogne ne participe pas personnellement au siège de Meaux (il est occupé en Picardie par les Dauphinois du Crotoy). Par ailleurs, l'alliance anglaise divise le parti bourguignon: il y a ceux, comme Jean de Luxembourg, qui y adhèrent sans remord et viennent même à Meaux «a mesnie privée ", tandis que d'autres préfèrent s'abstenir, voire passer au Dauphin ${ }^{40}$. Dans ce contexte d'alliance tiède, les chroniqueurs bourguignons proches de l'événement tels que Jean de Wavrin, Enguerran de Monstrelet et l'auteur d'une chronique anonyme du règne de Charles VI sont à la fois bien informés et peu enclins à polémiquer sur une guerre qui n'est pas la leur, d'où la qualité de leur témoignage. Seule exception dans ce groupe de chroniques : le Livre des trahisons que sa rédaction tardive (1465) et son caractère partisan disqualifient aux yeux de l'historien.

Le récit des autres chroniqueurs est beaucoup plus allusif : aucun n'est capable de donner le contenu du traité d'avril 1422, tous proposent une version moins étoffée et beaucoup plus partisane des faits. Quand Jean de Wavrin rapporte 24 séquences sur les 30 qui composent le récit du siège de Meaux, le Religieux de Saint-Denis n'en fournit que 16, Jean Juvénal 15, le Journal 13, Robert Blondel 13, pour ne citer que les plus prolixes. En outre, les chroniques parisiennes et armagnacs (les secondes, très tardives, s'inspirant des premières) sont peu cohérentes : elles hésitent sur le nom du capitaine de Meaux entre Vaurus ou Guichard de Chissay; elles précisent que les Dauphinois abandonnent la Ville du fait de l'hostilité de la bourgeoisie locale, alors que Meaux a déjà résisté sept mois, preuve de la loyauté des habitants qui auraient eu maintes occasions de trahir; elles affirment que les Dauphinois se replient d'eux-mêmes dans la forteresse, mais que les Anglais parviennent néanmoins à capturer Vaurus et son lieutenant dans la ville à cette occasion (en réalité Jean de Rouves était lieutenant $d u$ bailli de Meaux et a été exécuté à Paris après la chute du château comme l'atteste Nicolas de Fauquembergue, tandis que Vaurus est mentionné dans le traité de reddition). Surtout elles donnent une vision outrageusement partisane des faits.

La palme revient à Robert Blondel, précepteur du fils de Charles VII : il évoque le siège de Meaux dans deux de ses œuvres, l'Oratio historialis en 1449 (qu'il traduira ensuite sous le titre Les Droits à la couronne de France) et la Reductio Normanie écrite après 1450. Ces écrits sont plus patriotiques qu'historiques, car l'auteur cherche surtout dans l'histoire des arguments pour prouver la perfidie des Anglais. Ainsi le siège de Meaux est-il à chaque fois rapporté dans un chapitre au titre éloquent : «De la cruauté et des sacrilèges du roi d'Angleterre » ou «La vengeance divine punit toujours ceux qui commettent des sacrilèges ». Il souligne alors l'impiété d'Henri V et plus généralement sa mauvaise conduite lors du siège de Meaux : ce dernier va contre les usages de la 
guerre en conservant tout le butin sans le distribuer à ses soldats et en exilant injustement les 300 épouses nobles de ses prisonniers, mais il fait aussi preuve d'impiété en projetant d'emporter en Angleterre les reliques de saint Fiacre qui se trouvaient à Meaux (le mensonge est manifeste, car Henri V précise bien dans le traité que «all the reliques (...) and other goods mebles beyng in the faid Market, longyng to churches [have] to bene restitute in her places of the Churches of the faid Market, and that without fraude »). Il est pour cela puni par Dieu qui le fait périr d'une atroce maladie peu après. Dans ce contexte il faut considérer que la proposition faite par Henri $\mathrm{V}$ au bâtard de Vaurus de joindre son parti ou de périr d'une mort ignominieuse, qui permet à Robert Blondel de mettre dans la bouche de ce dernier des paroles superbes par lesquelles il dit préférer la mort au déshonneur, relève de l'invention - ou plutôt du déplacement, car il semble que la proposition ait été faite à Guichard de Chissay, si on suit la version bourguignonne des faits. Il est tout à fait révélateur de ce processus de réécriture partisane de l'histoire que la réponse de Vaurus s'amplifie avec le temps : elle tient en une phrase dans l'Oratio/Droits de la couronnne, mais elle enfle en un long discours patriotique dans la Reductio Normanie postérieure.

Les chroniqueurs bourguignons sont donc les mieux informés sur le siège de Meaux, mais aucun ne rapporte l'épisode de la femme enceinte dévorée par les loups ! Il est possible que le sort des civils les indiffère, mais l'occasion était cependant trop belle de salir l'honneur de l'ennemi et la légitimité de sa guerre pour la laisser passer. S'ils ne la notent pas, c'est qu'ils n'en ont pas connaissance, alors pourtant qu'ils puisent leur information auprès des participants du siège. Même le Livre des trahisons de France ou le chroniqueur anglais Thomas d'Elmham, pourtant attachés à souligner la cruauté de Vaurus et sa juste exécution, ne l'évoquent pas. D'ailleurs, même parmi les chroniques parisiennes, le Journal est le seul à rapporter l'épisode, les autres chroniqueurs restant très imprécis sur les actes de barbarie qu'ils lui supposent. Il n'y a donc probablement jamais eu de femme enceinte livrée aux loups ${ }^{41}$.

Cela ne signifie pas que le bâtard de Vaurus n'ait pas procédé à des exécutions sommaires. Les chroniques bourguignonnes précisent qu'il pendait à son orme tous les Anglais et les Bourguignons qu'il pouvait attraper, ce qui peut se comprendre dans un contexte de guerre civile dans lequel l'adversaire est aussi un traître. Jean de Wavrin précise cependant qu'il pendait aussi à son arbre des Français. Il est probable que le capitaine de Meaux a pendu des civils. En cette période d'anarchie politique, les chefs militaires en garnison loin de la Loire ne pouvaient espérer un approvisionnement ou un financement par le Trésor royal, aussi vivaient-ils sur le pays en réquisitionnant les biens des paysans. Il a certainement fait quelques exemples avec des paysans récalcitrants, pris dans des conflits de loyautés et peu enclins à livrer leurs réserves. Toutefois, le crime attribué par le Bourgeois est aussi extraordinaire que la situation de ce capitaine armagnac est banale. Lorsqu'en 1441 et 1444 le Bourgeois s'étrangle d'indignation devant les déprédations des écorcheurs, l'échevinage, puis le roi lui-même répondent placidement : «il faut bien qu'ils vivent ${ }^{42}$.

Les chroniqueurs parisiens sont en revanche unanimes dans leur crainte de Vaurus. Même le Religieux de Saint-Denis, Pierre de Fénin et l'auteur anonyme d'une chronique abrégée, pourtant hostiles aux anglo-bourguignons, sont effrayés par sa cruauté supposée à l'égard des civils, d'où leur gêne manifeste à rapporter ses exploits guerriers. Les Parisiens sont cependant surtout informés de ce qui se passe dans la capitale - ils s'attardent sur le sort des prisonniers qu'ils voient passer par leur ville -, mais sont tributaires d'informations de seconde main pour tout le reste. Il semble donc 
que leur témoignage sur l'ogre de Meaux, renseigne avant tout sur les rumeurs qui courent à son sujet dans la région. Selon un processus classique, le manque d'information dans un contexte de guerre sans front conduit chacun à donner corps à ses angoisses en les projetant sur les maigres nouvelles disponibles. En ce sens, les rumeurs sont surtout l'expression des craintes de ceux qui les rapportent ${ }^{43}$. Or les Parisiens sont d'autant plus disposés à croire les récits les plus fous sur ce capitaine gascon qu'ils redoutaient les courses que la garnison de Meaux menait jusque sous les murs de Paris depuis 1418 : la peur d'être pris et rançonné a alimenté les fantasmes les plus terrifiants. La rumeur noircit la mémoire du bâtard de Vaurus tout comme celle de son contemporain Guillaume de Flavy, lui aussi qualifié de tyran et accusé de «pendre filles malgré tous ceulx quy en vouloient parler, les violer, faire morir gens sans pitié et les noyer $»^{44}$.

L'auteur du Journal ne ment probablement pas - on se convaincra de son honnêteté à la manière dont il rapporte en 1427 l'exécution de Sauvage de Frémainville, l'écuyer qui avait failli capturer le duc de Bedford ${ }^{45}$ - mais il donne une épure rhétorique parfaite aux rumeurs les plus sombres qui courent dans la ville. Cela n'exclut pas cependant un dessein moins naïf : la narration circonstanciée de cette rumeur a son utilité dans l'économie générale du récit, ce qui explique le soin qu'il met à la rapporter. L'anecdote a en effet toutes les apparences d'un exemplum qui répond parfaitement à la définition qu'en donne la typologie des sources du Moyen Âge «[d'] un récit bref donné comme véridique et destiné à être inséré dans un discours pour convaincre un auditoire par une leçon salutaire ${ }^{46}$, à ceci près qu'il s'agit d'un exemplum horribilis brandi à des fins politiques. La leçon est transparente : Vaurus a péri par où il a péché. Ici, la morale vient au secours d'un discours partisan. Il s'agit d'expliquer au lecteur pourquoi un capitaine de sang noble, qui a prouvé sa bravoure et s'est rendu de lui-même après une résistance héroïque, est exécuté ignominieusement et pourquoi les autres prisonniers sont si maltraités lors de leur captivité. Solution: «tous ceux de ladite garnison ensuivaient la cruauté des deux tyrans », les frères Vaurus. Il s'agit aussi d'expliquer comment les Parisiens peuvent accepter un roi anglais et lutter contre ceux qui le combattent. Réponse : en 1420, «les Armagnacs [étaient] plus acharnés à cruauté qu'oncques mais, et tuaient, pillaient, efforçaient, ardaient les églises et les gens dedans, filles grosses et enfants, bref ils faisaient tous les maux en tyrannie et en cruauté qui pussent être faits par diable ni par homme; par quoi il convint qu'on traitât au roi d'Angleterre ${ }^{47}$ ! Dessiner le portrait d'un tyran monstrueux, c'est préparer son légitime tyrannicide et justifier la collaboration avec ses ennemis. La cruauté du bâtard de Vaurus justifie le traité de Troyes de 1420 et souligne le rôle pacificateur d'Henri V.

La petite trentaine de chroniques rapportant le siège de Meaux se classe donc en quatre groupes qui se définissent par leur position dans le temps, dans l'espace et surtout par leur position politique :

- les chroniqueurs anglais se divisent en deux sous-groupes :

- les chroniques de Londres sont tardives et loin du théâtre des opérations, donc mal informées,

- les Vies d'Henri $V$ sont plus proches de l'événement et bien informées sur la geste royale (Titus Livius s'inspirant du pseudo-Elmham), mais très partisanes et s'intéressent peu à Vaurus ;

- les chroniqueurs bourguignons sont le plus souvent neutres et parfois très bien informés sur le siège de Meaux lorsqu'ils sont contemporains de l'événement 
comme Jean de Wavrin (les autres s'inspirant plus ou moins de lui), mais ne disent rien sur les atrocités commises par Vaurus ;

- les chroniqueurs parisiens sont tous terrifiés par les atrocités qu'on prête à Vaurus, mais varient dans leur traitement de l'information selon que la barbarie supposée du capitaine de Meaux les gêne ou non sur le plan politique :

- les auteurs favorables au duc de Bourgogne comme celui du Journal les détaillent,

- les autres les éludent comme le Religieux de Saint-Denis ;

- les chroniqueurs armagnacs puis humanistes écrivent longtemps après les faits ; ils puisent leur information dans les chroniques parisiennes, mais soulignent dans un esprit partisan la loyauté et l'héroïsme du bâtard de Vaurus.

Reste à comprendre pourquoi Henri V n'a pas fait grâce à un capitaine qui s'était rendu à lui ${ }^{48}$, et pourquoi il lui a infligé une exécution ignominieuse à Meaux alors que les autres suppliciés qui ont eu le droit à un procès et une exécution à Paris dans les règles, même si dans leur cas la procédure était très formelle ${ }^{49}$. L'analyse du dossier de chroniques permet d'établir le déroulement du siège de Meaux avec une relative précision. Il en ressort que l'exécution infâmante du capitaine s'explique par le désir d'Henri V de faire un exemple. Il a pour cela plusieurs bonnes raisons.

D'abord, l'héroïsme de la garnison de Meaux commandée par Vaurus lui a coûté très cher. Le roi d'Angleterre ne s'attendait pas à une telle résistance lorsqu'il mit le siège devant la ville en septembre 1421, et les assiégés l'ont obligé à passer l'hiver en campagne, alors que l'épidémie frappait son armée. Au coût humain s'ajoute le coût financier que l'on peut comparer à celui du second siège de Meaux, en 1439: l'armée française de 6.400 combattants qui fit le siège pendant 2 mois coûta 100.000 1.t. au Trésor royal ${ }^{50}$, or Henri V dut maintenir en 1421-1422 une armée de 2.600 hommes pendant 9 mois $^{51}$.

Le roi d'Angleterre a aussi des raisons personnelles de vouloir se venger. Les sources bourguignonnes, ainsi que Pierre de Fénin, soulignent l'affront mortel fait à Henri V lorsque les assiégés firent monter sur les remparts un âne pelé qu'ils frappèrent pour le faire braire, en disant aux Anglais de venir délivrer leur roi qui les appellait au secours. Le Journal se fait aussi l'écho, à l'occasion du siège d'Ivry en 1424, d'un de ces rituels de dérision par lesquels des assiégés ou les assiégeants insultaient leur adversaire, mais si ce type de provocation était courant, il n'est pas banal, car Henri V en conçut une violente rancune contre la garnison de Meaux. L'âne, acteur privilégié de ce genre d'outrage, symbolisait en effet le travail pénible, la saleté, l'animalité, la bêtise et le priapisme ${ }^{52}$. Or le roi d'Angleterre, plutôt magnanime avec les vaincus, était intraitable avec ceux qui attentaient à son honneur, comme ces canonniers qui avaient visé sa tente à Louviers lors d'une entrevue diplomatique, ou les soldats de Rouen qui l'avaient insultés comme ceux de Meaux ${ }^{53}$.

Henri $\mathrm{V}$ a enfin des raisons stratégiques de faire un exemple. Après le succès du traité de Troyes, le roi était retourné en Angleterre, mais la situation s'est détériorée pour ses lieutenants sur le continent: en mars 1421 le duc de Clarence est écrasé à Baugé et les troupes du Dauphin marchent sur Paris; Henri V revient donc précipitamment en juillet et contre-attaque. Il prend Dreux, Épernon, Nogent-le-Roi, Senlis. C'est dans ce contexte qu'il met le siège devant Meaux, l'une des principales forteresses franciliennes, qui contrôle en outre l'approvisionnement de Paris par la Marne. Vaincre et châtier durement Meaux, c'est briser d'avance la résistance de places 
moins fortes. Les chroniqueurs bourguignons, mais aussi Pierre de Fénin, le Religieux de Saint-Denis et Jean Juvénal soulignent le succès de cette stratégie d'intimidation: une quinzaine de forteresses se rendirent ensuite au roi d'Angleterre.

Pour les Bourguignons, pas de doute, Henri V se venge sur les assiégés après cette victoire difficile. Sa vengeance passe par l'exécution de ceux qui l'ont offensé, et le bâtard de Vaurus n'est pas le seul concerné. Le second article du traité donne une liste de 13 membres de la garnison de Meaux devant se rendre à merci au roi d'Angleterre (qui promet en revanche la vie sauve aux autres); sur les 13, cinq sont exécutés, cinq sont épargnés parce qu'ils serviront de monnaie d'échange pour obtenir la reddition d'autres forteresses qui dépendent d'eux, mais les trois derniers ont aussi la vie sauve, sans raison apparente. En fait, seuls les responsables de la résistance et de l'affront fait à Henri $\mathrm{V}$ ont été châtiés : le bâtard de Vaurus en tant que capitaine général (ainsi que son frère auquel il est souvent associé), mais aussi le bailli de Meaux, son lieutenant, plus un soldat qui s'était moqué du roi d'Angleterre sur les remparts. Cinq chroniques bourguignonnes sur six évoquent la pendaison du bâtard de Vaurus à « son arbre » et toutes l'expliquent encore une fois par une vengeance d'Henri V « parce que ledit bastard avoit au temps passé et par long espace fait pendre audit arbre plusieurs Anglois et Bourgongnons. Sy en prist maintenant le roy anglois vengeance pour tout le temps passé ${ }^{54}$. L'âpreté des combats, les insultes proférées par les assiégés et une justice de guerre expéditive suffisent à expliquer la justice d'exception exercée à l'encontre du chef de la garnison de Meaux, sans qu'il soit besoin de lui attribuer un crime atroce.

Voilà donc un fait divers passé au rang de fantasme de chroniqueur terrifié par la crise de l'ordre public. Il est très rare de pouvoir l'établir, mais en l'occurrence il semble bien que le récit du Journal d'un bourgeois de Paris soit un exemplum horribilis saturé de clichés dont la puissance est encore assez forte pour égarer les historiens. On ne peut que souligner ici la fragilité des témoignages des chroniqueurs pour établir la réalité des événements passés. Ces sources égarent d'autant plus l'historien que leur valeur littéraire, donc leur capacité de séduction, est grande. Le dossier du siège de Meaux souligne la nécessité d'une critique serrée des chroniques pour établir les faits, mais il montre aussi l'intérêt des déformations de l'information pour l'histoire culturelle et l'étude des mentalités.

Le résultat de cette petite enquête un peu érudite serait bien anecdotique, s'il ne venait ajouter un élément de plus au volumineux dossier des fabulations de chroniqueurs médiévaux enclins à délégitimer la violence de l'adversaire en en soulignant l'horreur. La relativisation récente de la violence de la fin du Moyen Âge était passée jusqu'à présent par l'identification et le repérage d'accusations stéréotypées - ainsi attribue-t-on très souvent aux ennemis ou aux brigands une litanie de crimes aussi irrémissibles qu'imprécis : incendies, sacrilèges, viols, rapts, vols, meurtres ${ }^{55}$. Les intellectuels et les chroniqueurs de tous bords les colportent, avant qu'ils soient remployés dans la propagande politique. La circulation sans fin de ces stéréotypes leur donne réalité pour les contemporains, qui ne les évoquent pas sans terreur, mais elle les décrédibilise pour l'historien. Le fait que cette liste de crimes irrémissible soit formalisée dès le début de la guerre de Cent Ans suggère d'ailleurs qu'elle est un effet des angoisses suscitées par chaque crise de l'ordre public, bien plus que d'un long processus s'ensauvagement de la société ${ }^{56}$. Cette violence stéréotypée contraste avec la violence ordinaire, telle qu'elle ressort de l'étude de milliers de lettres de rémission, par 
lesquelles un sujet du roi de France craignant d'être inquiété par la justice supplie le roi de lui accorder sa grâce. Cette source montre que seulement $1 \%$ des crimes pardonnés relèvent de la présence anglaise à l'échelle du siècle, tandis que la majorité des crimes sont perpétrés par des hommes ordinaires, bien insérés dans la société, à la suite une rixte-homicide provoquée par une insulte $(57 \% \text { des cas })^{57}$. Par ailleurs, l'étude du vol et $\mathrm{du}$ brigandage vient confirmer la place limitée des marginaux et des criminels professionnels dans la violence médiévale, car la majorité des condamnés sont aussi des hommes bien intégrés, appartenant au monde de l'artisanat et du salariat urbain ou rural qui, pour être modeste, n'est pas miséreux, tandis que la haine ou la jalousie suscitent au moins autant de vols que le lucre ${ }^{58}$.

L'autre intérêt du dossier de chroniques autour du siège de Meaux est de montrer que même des anecdotes détaillées, dont la précision pourrait être un gage de véracité, peuvent aussi être le fruit de la peur. Si l'infamie du bâtard de Vaurus est la formalisation littéraire et partisane d'une rumeur, que dire alors des Jacques violeurs et anthropophages décrits par des chroniqueurs aussi aristocratiques de Jean Le Bel et Froissart $^{59}$ ? Que dire aussi des méfaits reprochés aux Tuchins du Languedoc par le Religieux de Saint-Denis dans les années 1380, alors que leur mouvement de résistance aux brigands se trouve délégitimé par les trêves avec les Anglais et la reprise en main du royaume par Charles VI ${ }^{60}$ ? Ces dossiers mériteraient d'être réexaminés.

La relativisation des violences guerrières permet donc de mieux comprendre les « déchaînements de la guerre civile » évoqués en introduction. Nul doute que la guerre de Cent Ans a dû s'accompagner de débordements tragiques, mais il est non moins certain que ceux-ci ne furent pas aussi systématiques que ce que laissent penser les références stéréotypées aux violences commises par les gens d'armes et les anecdotes circonstanciées rapportées par les contemporains. La fin du Moyen Âge n'est pas un âge de barbarie ordinaire, c'est une époque traversée par de grandes peurs liées à des troubles répétés de l'ordre public provoqués par la maturation accélérée des Etats monarchiques. Le choc des souverainetés entre les rois d'Angleterre et de France pour la Guyenne mène à la guerre et la guerre entraîne des troubles et une crise de la légitimité politique (donc de la violence légitime) qui suscitent une grande angoisse dans la population, qui voit désormais des ogres partout.

\section{Annexe 1: les maréchaux de France envoient au trésorier des guerres une montre du bâtard de Vaurus (26/8/1419)}

$\mathrm{BnF}, \mathrm{PO}, 2946, \mathrm{n}^{\circ}$ 65445, dossier Vauruz (le document est hélas l'unique pièce du dossier) :

«Les mareschaux de France a nostre amé Hemon Raguier, tresorier des guerres du roy nostre sire ou a son lieutenant salut. Nous vous envoyons atachee a ces presentes sous le scel commun de la mareschaucee de France la reveue du Bastart de Vauruz, escuier, et de dix-neuf autres escuiers de sa chambre receuz a Melun le XXVI ${ }^{\text {eme }}$ jour d'aoust l'an mil quatre cens dix neuf, souffisamment montez et armez pour servir le roy nostre dit seigneur et monseigneur le regent le royaulme daulphin de Viennois en leurs presentes guerres contre les Angloys leurs anciens ennemis et partout ailleurs où il plaira aux dits seigneurs ordonner en la compagnie de messire Arnault de Barbazan, conseiller et premier chambellan de mon dit seigneur le regent et de messire Tanguy du Chastel, conseiller et mareschal des guerres de mon dit seigneur le regent, chevalier, de certain 
nombre de gens d'armes et de trait a eulx ordonné pour ladicte cause soubz le gouvernement de mon dit seigneur le regent; si vous mandons que au dit Bastart des gaiges de luy et des autres contenuz en ladicte reveue vous faictes compte, prest et payement en la maniere acoustumee et comme il appartient. Donné audit lieu soubz le seel l'an et jour dessusdiz. Landes ».

Annexe 2 - Les sources sur le siège de Meaux (1421-1422) :

\section{Chroniques bourguignonnes}

\begin{tabular}{|l|l|l|}
\hline $\mathrm{N}^{\circ}$ & \multicolumn{1}{|c|}{} & DATE \\
\hline $\mathrm{A}$ & $\begin{array}{l}\text { Recueil des croniques et anchiennes istories de la Grant Bretaigne, à } \\
\text { présent nommé Engleterre par Jehan de Waurin, seigneur du } \\
\text { Forestel, éd. William Hardy, London, 1868, II, pp. 385-406 }\end{array}$ & 1445 \\
\hline $\mathrm{B}$ & $\begin{array}{l}\text { La chronique d'Enguerran de Monstrelet, éd. Louis Douët-d'Arcq, } \\
\text { SHF, Paris, 1857, IV, pp. 70-98 }\end{array}$ & $<1453$ \\
\hline $\mathrm{C}$ & $\begin{array}{l}\text { Chronique anonyme du règne de Charles VI, éd. L. Douët-d'Arcq in } \\
\text { La Chronique d'Enguerran de Monstrelet, éd. Louis Douët-d'Arcq, } \\
\text { SHF, Paris, V, pp. 305-306 }\end{array}$ & $<1450$ \\
\hline $\mathrm{D}$ & $\begin{array}{l}\text { Chronique normande de Pierre Cochon, notaire apostolique à Rouen, } \\
\text { éd. Charles de Robillard de Beaurepaire, Rouen, 1870, pp. 287-288 }\end{array}$ & $<1456$ \\
\hline $\mathrm{E}$ & $\begin{array}{l}\text { Chronique de Jean Le Fèvre, seigneur de Saint-Rémy, éd. François } \\
\text { Morand, SHF, Paris, 1881, II, pp. 44-56 }\end{array}$ & $<1468$ \\
\hline $\mathrm{F}$ & $\begin{array}{l}\text { Euvres de Georges Chastellain, éd. Kervyn de Lettenhove, 1863, I, } \\
\text { pp. 282-284 et 294-308 }\end{array}$ & $<1475$ \\
\hline $\mathrm{G}$ & $\begin{array}{l}\text { Le Livre des trahisons de France, éd. Kervyn de Lettenhove, in } \\
\text { Chroniques relatives à l'histoire de la Belgique sous la domination } \\
\text { des ducs de Bourgogne, 1878, II, pp. 167-168 }\end{array}$ & $\sim 1465$ \\
\hline
\end{tabular}

Chroniques anglaises

\begin{tabular}{|l|l|l|}
\hline $\mathrm{N}^{\circ}$ & VIES D'HENRI V & DATE \\
\hline $\mathrm{H}$ & $\begin{array}{l}\text { Thomas d'Elmham, Vita et gesta Henrici quinti, Anglorum regis, ed. } \\
\text { Thomas Hearne, Oxford, 1727, pp. 315-329. }\end{array}$ & $<1440$ \\
\hline $\mathrm{H} 1$ & $\begin{array}{l}\text { Titus Livius Forojuliensis, Vita Henrici Quinti, ed. Thomas Hearne, } \\
\text { Oxford, 1716. }\end{array}$ & $<1450$ \\
\hline $\mathrm{N}^{\circ}$ & CHRONIQUES DE LONDRES & DATE \\
\hline $\mathrm{H} 2$ & $\begin{array}{l}\text { The Great Chronicle of London, ed. By A. H. Thomas and I. D. } \\
\text { Thorney, London, 1938, p. 120-122. }\end{array}$ & $<1450$ \\
\hline $\mathrm{H} 3$ & $\begin{array}{l}\text { Ms. Julius B II, } \\
\text { Cleopatra C IV, } \\
\text { Vitellius A XVI, in The chronicles of London, ed. C. L. Kingsford, } \\
\text { Oxford, 1905, p. 74, 128, 271. }\end{array}$ & 1496 \\
\hline H4 & $\begin{array}{l}\text { A short English Chronicle, ed. by James Gairdner, J. Stow, Three } \\
\text { fifteenth century chronicles, Camden Society, New Series, XXVIII, } \\
1880, \text { p. 57-58. }\end{array}$ & 1446 \\
\hline H5 & $\begin{array}{l}\text { A Northern Chronicle (1399-1430), in C. L. Kingsford, English } \\
\text { Historical Litterature in the Fifteenth Century, p. 290. }\end{array}$ & $\sim 1450$ \\
\hline H6 & The Latin Brut, in C. L. Kingsford, English Historical Literature in & $<1437$ \\
\hline
\end{tabular}




\begin{tabular}{|l|l|l|}
\hline & the Fifteenth Century, p. 336. & \\
\hline H7 & $\begin{array}{l}\text { London chronicle for 1421-1430, in C. L. Kingsford, English } \\
\text { Historical Litterature in the Fifteenth Century, p. 295. }\end{array}$ & $\sim 1450$ \\
\hline
\end{tabular}

Chroniques parisiennes

\begin{tabular}{|l|l|l|}
\hline $\mathrm{N}^{\circ}$ & FAVORABLES AUX ANGLO-BOURGUIGNONS & DATE \\
\hline $\mathrm{I}$ & $\begin{array}{l}\text { Journal d'un bourgeois de Paris, éd. C. Beaune, Paris, 1990, § 324, } \\
329,333-336,340,345,347 .\end{array}$ & $<1449$ \\
\hline $\mathrm{J}$ & $\begin{array}{l}\text { Journal de Clément de Fauquembergue, greffier du parlement de } \\
\text { Paris, éd. Alexandre Tuetey et Henri Lacaille, SHF, Paris, 1903-1915, } \\
\text { II, pp. 39-49. }\end{array}$ & $<1435$ \\
\hline $\mathrm{N}^{\circ}$ & FAVORABLES AU DAUPHIN & DATE \\
\hline $\mathrm{K}$ & $\begin{array}{l}\text { Mémoires de Pierre de Fénin, éd. Claude-Bernard Petitot, Paris, 1825, } \\
\text { VII, pp. 352-356. }\end{array}$ & $<1433$ \\
\hline $\mathrm{L}$ & $\begin{array}{l}\text { Chronique du religieux de Saint-Denys, éd. Louis Bellaguet, Paris, } \\
\text { 1994, VI, pp. 449-455. }\end{array}$ & $<1421$ \\
\hline $\mathrm{M}$ & $\begin{array}{l}\text { Fragment d'une version française des chroniques de Saint-Denis, éd. } \\
\text { A. Vallet de Viriville, in Chronique de Charles VII de Jean Chartier, } \\
\text { Paris, 1858, III, pp. 248-251. }\end{array}$ & $<1460$ \\
\hline
\end{tabular}

\section{Chroniques armagnacs}

\begin{tabular}{|l|l|l|}
\hline $\mathrm{N}^{\circ}$ & CHRONIQUES DU XVE SIECLE & DATE \\
\hline $\mathrm{N}$ & $\begin{array}{l}\text { Histoire de Charles VI par Jean Juvénal des Ursins, dans Le livre des } \\
\text { fais et bonnes meurs du sage roy Charles V par Christine de Pisan, } \\
\text { éd. J.F. Michaud et J.J.F. Poujoulat, Paris, 1836, pp. 562-564. }\end{array}$ & $<1470$ \\
\hline $\mathrm{O}$ & $\begin{array}{l}\text { Robert Blondel, Reductio Normanie : ouvres de Robert Blondel, éd. } \\
\text { Alexandre Héron, Rouen, 1892, I, pp. 198-199. }\end{array}$ & $\sim 1450$ \\
\hline $\mathrm{P}$ & $\begin{array}{l}\text { Robert Blondel, Des droits à la couronne de France : aeuvres de } \\
\text { Robert Blondel, éd. Alexandre Héron, Rouen, 1892, I, pp. 364-365. }\end{array}$ & $\sim 1450$ \\
\hline $\mathrm{Q}$ & $\begin{array}{l}\text { Chronique de Jean Raoulet, éd. Auguste Vallet de Viriville, dans } \\
\text { Chronique de Charles VII de Jean Chartier, Paris, 1858, III, p. 170. }\end{array}$ & $<1467$ \\
\hline $\mathrm{R}$ & $\begin{array}{l}\text { Les Chroniques du roi Charles VII par Gilles Le Bouvier (dit le le } \\
\text { héraut Berry), éd. Henri Courteault et Léonce Celier, Marie-Henriette } \\
\text { Jullien de Pommerol, SHF, Paris, 1979, pp. 98-99. }\end{array}$ & $<1455$ \\
\hline $\mathrm{N}^{\circ}$ & CHRONIQUES HUMANISTES & DATE \\
\hline $\mathrm{S}$ & $\begin{array}{l}\text { Nicolas Gilles, Les Annales et chroniques de France, Paris, Jean } \\
\text { Macé, 1553, p. 66. }\end{array}$ & $<1500$ \\
\hline $\mathrm{T}$ & $\begin{array}{l}\text { Robert Gaguin, Les Chroniques, excellents faits et virtueux gestes de } \\
\text { très illustres très chrétiens et magnanimes et victorieux roys de } \\
\text { France, Paris, Galiot du Pré, 1515, p. 154. }\end{array}$ & $<1501$ \\
\hline
\end{tabular}

Annexe 3 : Les séquences du siège de Meaux:

1. Description de la double forteresse de Meaux : Ville et Marché $(\mathrm{H})$ 
2. Septembre 1421 : Henri V fait préparer des engins de siège à Lagny (A, B, C, E, F, K, L, M, N, O, P, Q, R, S, T).

3. envoie le duc d'Exeter prendre possession des faubourgs de Meaux (A, B, C, E, F).

4. Octobre 1421: Henri $\mathrm{V}$ arrive et établit un camp fortifié devt Meaux avec 20.000 hommes (A, B, C, D, E, F, J).

5. Description du dispositif anglais encerclant Meaux $(\mathrm{H})$.

6. Les assiégés résistent avec vaillance pendant 7 mois (A, D, F, H, J, L, N, S).

7. Le sire de Cornouaille est blessé durant le siège et y perd son fils, si bien qu'il renonce à combattre et retourne en Angleterre (A, B, D, E, F, K, L, M, N, Q).

8. Ville et Marchés tenus par des gens du Dauphin : le bâtard de Vaurus capitaine général, Denis de Vaurus son frère, Perron de Luppé, Guichard de Chissay, Philippe Malet, Louis Gast, Le Borgne de Chauquin, Jean d'Aulnay, Tromagon, Bernard de Mereville, Philippe de Gamaches et 1.000 hommes d'armes aguerris sans compter les bourgeois (A, B, C, E, H, K, L, N, R).

9. Jean de Luxembourg vient a privee maisnie pour obtenir la délivrance de son frère le comte de Conversan prisonnier de Peron Luppé dans Meaux (A, F).

10. Arthur de Bretagne libéré, vient assister Henri V à Meaux (F).

11. Les assiégés insultent Henri V avec un âne sur les remparts (A, B, E, F, H, K).

12. Echec de la tentative de secours du sire d'Offémont, venu pour prendre le commandement de la place avec 40 hommes (A, C, E, F, H, I, J, K, L, M, N, R, T).

13. Faute de ce renfort, la garnison ne peut plus tenir les remparts de la Ville et se retranche dans le Marché (A, E, F, I, K, L, M, N, P, R, T).

14. Jean de Guigny, savoyard, s'en rend compte et lance un assaut aisé (A, E, F, I, J, $\mathrm{M}, \mathrm{P})$.

15. Henri $\mathrm{V}$ prend ensuite une île où il installe son artillerie, qui ruine les murailles de Meaux (A, B, E, F, H, I, K, P).

16. Les machines de guerre anglaises font merveille $(\mathrm{H})$.

17. Assiégés refusent obstinément de se rendre (A, B, E, F, K).

18. Assiégés résistent à un assaut mémorable de 8 heures $(\mathrm{A}, \mathrm{B}, \mathrm{E}, \mathrm{F}, \mathrm{K})$.

19. Assiégés en sont réduits à se servir de tournebroches comme lances $(\mathrm{A}, \mathrm{B}, \mathrm{E}, \mathrm{F}$, $\mathrm{K})$.

20. Guichard de Chissay s'illustre par sa vaillance si bien qu'après la reddition de la garnison, Henri V veut se l'attacher, mais il reste fidèle au Dauphin (A, B, F, M).

21. Jean de Guigny et le Bâtard de Thien s'étant illustrés par leur vaillance sont faits chevaliers par Henri V (A, B, C, F).

22. Les Anglais prennent les moulins du Marché, ce qui prive les assiégés de farine (A, C, E, F, H, L, M, N).

23. Fin avril 1422, les assiégés commencent à négocier par l'intermédiaire de Philippe Mallet, Péron de Luppé, Jean d'Aulnay, Sivador de Girêmes, Le Borgne de Cauchy, Jean de L'Espinasse, Guillaume du Fossé, Olivier de Mainozit (A, B, C, D, E, F, I, K, L, M, N, O, P, Q, R, S, T).

24. Le traité stipule (confirmé d'après l'original ou ajouté d'après celui-ci) [A, B, C, E, F, H, H2]

a. Reddition le $10 / 5$ 
b. Sont livrés à la justice d'Henri V : les frères Vaurus, Louis Gast, Jean de $\underline{\text { Rouvères, }}$ Tromagon, Bernard de Mereville, Jean de La Motte et Orace qui avait sonné du cors par dérision, Guichard de Chissay, Péron de Luppé, Robert de Girêmes, Philippe de Gamaches, Jean d'Aulnay.

c. Les frères Vaurus, Louis Gast, Jean de Rouvères devront être jugés.

d. Demeureront prisonniers tant que leur forteresses ne sont pas rendues : Guichard de Chissay, Péron de Luppé, Robert de Girêmes, Philippe de Gamaches, Jean d'Aulnay

e. Demeurent à merci : les canonniers, les coupables de la mort de Jean sans Peur, les sujets du roi d'Angleterre et ceux qui ont juré la paix finale en 1420 .

f. Les autres hommes d'armes et les gens du marché ont la vie sauve mais seront rançonnés.

g. Le prisonnier de Péron de Luppé est quitte de rançon.

h. Livraison des richesses du Marché.

i. Prisonniers de Meaux délivrés.

j. Interdiction de fuir pendant la trêve.

k. Désignation de 24 otages, garants du traité

1. 100 parmi les plus notables doivent s'engager par écrit à respecter le traité

m. Trêve jusqu'au 10/5

25. 700 à 800 hommes d'armes se rendent. Les plus importants sont envoyés en Angleterre, le reste est emprisonné à Paris et ailleurs [A, B, C, E, F, H, H7, I, J, $\mathrm{L}, \mathrm{M}, \mathrm{N}, \mathrm{O}, \mathrm{T}]$.

26. Henri V se venge en faisant décapiter le bâtard de Vaurus, général capitaine de la place, et le fait pendre à l'arbre où il avait l'habitude d'exécuter Anglais, Bourguignons et Français [A, B, C, E, F, H, I, K, L, M, N, O, Q, R, T].

27. Les biens du Marché sont distribués au plaisir d'Henri V [A, B, E, K, H, L, M, $\mathrm{N}, \mathrm{P}]$.

28. Les responsables politiques locaux sont décapités aux Halles (Denis de Vaurus, le bailli Louis Gast, son lieutenant maître Jean de Rouves) et Orace qui avait insulté Henri V [A, B, C, E, F, H, I, J, L, N].

29. La reddition de Meaux entraîne celle de nombreuses places : Crépy-en-Valois, Pierrefonds, Merlau, Offémont, Compiègne, Gournay-sur-Aronde, Cressonsac, Mortemer, Neufville-en-Hez, Gamaches, Montaigu, Moy en Laonnois, Montescourt, Brassy [B, E, F, H, K, L, N].

30. Henri $\mathrm{V}$ restaure la forteresse de Meaux et y installe une garnison [A, B, F, K, $\mathrm{M}]$.

\footnotetext{
${ }^{1}$ Voir par exemple B. Geremek, Les marginaux parisiens aux XIVe et XVe siècles, Paris, 1976 ; G. Bois, Crise du féodalisme. Economie rurale en Normandie du début du XIV siècle au milieu du XVI siècle, Paris, 1976, rééd. 1981 ; T. H. Aston, C. H.E. Philpin (éd.), The Brenner Debate. Agrarian Class Structure and Economic Development in Pre-industrial Europe, Oxford, 1985. Pour un état récent de l'historiographie de la fin du Moyen Âge en France, voir B. Bove, Le temps de la guerre de Cent Ans, Paris, Belin, 2009, p. 507-540.

${ }^{2} \mathrm{G}$. Bois, La grande dépression médiévale : XIV $-X V^{e}$ siècles. Le précédent d'une crise systémique, Paris, 2000, p. 83.
} 
${ }^{3}$ De Jules Michelet à Christopher Allmand (par ex. J. Michelet, Jeanne d'Arc, 1856 rééd. Paris, 1888, p. xxxiii-lx, à comparer avec le Journal, § 239 ; C. Allmand, «War and non-combatants in Middle Ages », in M. Keen (dir.), Medieval Warfare. A History, Oxford, 1999, p. 265-266).

${ }^{4}$ C. Beaune, «La rumeur dans le Journal du Bourgeois de Paris », in La circulation des nouvelles au Moyen Âge, Paris, 1994, p. 191-203 ; C. Gauvard, «Rumeur et stéréotypes à la fin du Moyen Âge », Ibid., p. 157-177.

${ }^{5}$ Comme celle utilisée pour la prise de Soissons en 1414 : «plusieurs furent pendus, et les femmes de religion et les autres prudes femmes et bonnes pucelles efforcées, et tous les hommes rançonnés, et les petits enfants, et les églises et reliques pillées (...). On n'ouït oncques parler que les Sarrasins firent pis » (Journal, § 100 ; voir aussi notes 59 et 60). C. Beaune, « La rumeur », p. 198.

${ }^{6}$ On s'appuiera ici sur la dernière édition de cette œuvre : Journal d'un bourgeois de Paris de 1405 à 1449, éd. Colette Beaune, Paris, Le Livre de Poche, 1990.

${ }^{7}$ Journal, p. 11-18 ; C. Allmand, The Hundred Years War, p. 207 de l'édition française (Paris, 1989).

${ }^{8}$ Journal, $\$ 343,344,345$.

${ }^{9}$ Ainsi le jésuite G. Daniel, Histoire de France depuis l'établissement de la monarchie françoise dans les Gaules, Amsterdam, 1720, p. 912, C. Villaret, Histoire de France, depuis l'établissement de la monarchie jusqu'à Louis XIV, Paris, 1770, VII, p. 293 ou N. Baudot de Juilly, Histoire et règne de Charles VI, Paris, 1753, pp. 320-351.

10 J. C. L. Sismonde de Sismondi, Histoire des Français, Bruxelles, 1837, VIII, p. 429 ; P. Brugière de Barante, Histoire des ducs de Bourgogne de la maison de Valois, Bruxelles, 1838, I, pp. 422-423.

${ }^{11}$ Anonyme, «Déclaration d'amour à Madame... Y », La Chronique de Champagne, 1837, n¹, pp. 417433.

${ }^{12}$ H. Duval-Pineu, Histoire de France sous le règne de Charles VI, 1842, II, pp. 369-370.

13 «L'assassinat du grand prince Luchin Visconti par sa femme Isabelle de Fiesque (1349) vaut mieux que l'orme de Vaurus » (Stendhal, Rome, Naples et Florence, Paris, 1826, I, p. 99).

${ }^{14}$ J. H. Wylie, The reign of Henry the Fifth, III, Cambridge, 1929, III, p. 352. J. Berlioz, Catastrophes naturelles et calamités au Moyen Âge, Lausanne, 1998, pp. 27-38.

15 C. Beaune, «La rumeur», p. 198-199. Elle fonde son intuition sur le témoignage positif des chroniqueurs armagnacs qui soulignent la loyauté héroïque du bâtard de Vaurus au parti « français », mais ces chroniques sont insuffisantes pour établir la vérité car elles sont aussi tardives que patiales (C. Beaune, Naissance de la nation France, Paris, 1985, pp. 449-450).

${ }^{16}$ Document édité par A. Vallet de Viriville, Chronique de Charles VII de Jean Chartier, Paris, 1858, III, p. 249 , n. 2.

${ }^{17}$ Bibliothèque nationale de France (désormais BnF), Pièces Originales, 2946, n 65445 , doss. Vauruz (annexe 1). Pour le document du 30/11/1419 : A. Vallet de Viriville, Histoire de Charles VII, roi de France, et de son époque (1404-1461), Paris, 1861, I, p. 191.

${ }^{18}$ B. Schnerb, Les Armagnacs et les Bourguignons, Paris, 1988, pp. 224-225.

${ }^{19}$ G. Dupont-Ferrier, Gallia Regia, Paris, 1954, IV, n ${ }^{\circ} 15081,15377$. A. Vallet de Viriville, Histoire de Charles VII, I, p. 191.

${ }^{20}$ Les références des chroniques relatives au siège de Meaux sont rassemblées dans l'annexe 2.

${ }^{21}$ Pas de famille gasconne de ce nom dans Anselme, Histoire de la maison royale de France et des grands officiers de la Couronne, Paris, 1674 ; G. Saffroy, Bibliographie généalogique, héraldique et nobiliaire de la France, Paris, 1974 ; A. La Chesnaye-Desbois, Dictionnaire de la noblesse, Paris, 1876.

${ }^{22}$ Aucune trace au Parlement (AN : X1a 48 à 68, X1a 9190 à 9196 et X2a 15-17), dans le Trésor des chartes (AN : JJ 1 à 264). Rien dans D. F. Secousse et alii, Ordonnances des roys de France, Paris, 17291734. Aucune trace de sceau, en particulier dans G. Demay, Inventaire de la collection Clairembault, Paris, 1886 ; L. Douët-d'Arcq, Collection de sceaux aux Archives nationales, Paris, 1863 ; P. La Plagne Barris, Sceaux gascons du Moyen Âge, Paris, 1889 ; P. Raymond, Sceaux des Archives du département des Basses-Pyrénées, Pau, 1874 ; P. de Bosredon, Sigillographie du Périgord, Périgueux, 1880.

${ }^{23}$ Référence en annexe.

${ }^{24}$ J. Michelet, Histoire de France, Paris, 1861, IV, p. 340 et Précis d'histoire de France, Paris, 1835, p. 134.

${ }^{25}$ C. Gauvard, «Rumeur et stéréotypes », p. 176.

${ }^{26}$ Journal, § 187, 220, 256, 262, 276, 322, 328, 376, 378, 381, 481, 524, 579, 612.

27 J. Brosse, Dictionnaire des arbres de France. Histoire et légendes, Paris, 2002, p. 133-136. L'orme de justice est archaïque au $\mathrm{XV}^{\mathrm{e}}$ siècle, mais le souvenir de sa fonction est toujours vivace pour l'auteur du 
Journal puisqu'il écrit plus loin que «le roi de France était le droit orme aux larrons de chrétienté », c'est-à-dire ferme justicier de tous les criminels (Journal, § 792).

${ }^{28}$ C. Gauvard, Violence et ordre public au Moyen Âge, Paris, 2005, p. 210. Le meurtre de la femme enceinte est une transgression souvent mise en avant par les chroniqueurs ou leurs illustrateurs pour souligner l'illégitimité des actions politiques ou militaires qui y conduisent, ainsi le Bourgeois en 1418 et 1420 (Journal, $\S 224,276$ ) et l'illustrateur des Vigiles de Charles VII de Martial d'Auvergne pour les révoltes de 1413 et $1418(\mathrm{BnF}, \mathrm{Fr} .5054$, fol. 8v, 16v)

${ }^{29}$ Jacques de Voragine, La Légende dorée, éd. A. Boureau (dir.), Paris, 2004, pp. 458-459.

${ }^{30}$ J. M. Moriceau, Histoire du méchant loup. 3000 attaques sur l'homme en France, XVe-XXe siècle, Paris, 2007, p. 19-29.

${ }^{31}$ Journal, $§ 772$. Gaston Fébus assure que « le loup aime la chair humaine et peut-être s'il était plus fort n'en mangerait-il pas d'autre » (Le Livre de la chasse, éd et trad. R. et A. Bossuat, Paris, 1986, p. 64-66).

${ }^{32}$ Par exemple chez Nicolas de Clamanges (C. Gauvard, "De grace especial ». Crime, Etat et société en France à la fin du Moyen Âge, Paris, 1991, p. 212).

${ }^{33}$ Le folklore comme le Livre de la chasse de Gaston Fébus attestent la croyance dans l'endocanibalisme du loup, les prétendants éconduits par la louve se vengeant en dévorant celui qui l'a couverte (S. Bobbé, L'ours et le loup. Essai d'anthropologie symbolique, Paris, 2002, p. 34).

${ }^{34}$ L. Hablot, La Devise, mise en scène du prince, mise en scène du pouvoir, II, Devisier, thèse de l'université de Poitiers, 2001, p. 533. On peut s'interroger sur le choix comme devise du duc d'Orléans de ce prédateur peu valorisant. Il s'explique probablement par l'homophonie entre « loup » (associé à l'âme «il est ») et «Louis », tandis que la devise figure un loup colleté d'une clochette, c'est-à-dire un loup domestique et courtois, comme on en trouve dans la tapisserie de la Dame à la Licorne représentant le toucher (A. Erlande-Brandenburg, La dame à la licorne, Paris, 1989, p. 51).

${ }^{35}$ On voit sur le frontispice des manuscrits de la justification de l'assassinat de Louis d'Orléans par Jean Petit un lion donnant un coup de patte à un loup ravissant la couronne de France (BnF, Fr. 5733, fol. 2v ; Musée Condé, Ms. 878, fol. 2 ; Vienne, Osterreischische Nationalbibliothek, cod. 2657, fol. 1v).

${ }^{36}$ Ainsi Philippe de Morvilliers (Journal, § 325). Voir aussi Christine de Pisan dans son Livre de paix (C. Gauvard, «De grace especial », p. 225) ou Eustache Deschamps (CEuvres complètes d'Eustache Deschamps, éd. Le Queux de Saint-Hilaire, Paris, 1878, I, p. 309). C'est un motif rhétorique courant que l'on retrouve ensuite chez Robert Gobin, Les Loups ravissans, cestuy livre ou autrement doctrinal moral intitulé est, Paris, vers 1503, dans lequel le diable donne des leçons à des loups ; en face de lui, sainte Doctrine accuse les loups de persécuter les brebis. Cet ouvrage s'inscrit dans un courant réformateur de sensibilité apocalyptique : l'auteur y dépeint une extension du Mal, sous forme d'une lycanthropisation de la société, qui prélude à l'avènement du Christ.

${ }^{37}$ Journal, § 262, 271 et 16, 100, 102, 194, 256, 325, 328, 329, 376, 612, 633, 760.

${ }^{38}$ C. Lethbridge Kingsford, English Historical Litterature in the Fifteenth Century, Oxford, 1913, p. 51 et suiv.; D. Rundle, "The Unoriginality of Tito Livio Frulovisi's Vita Henrici Quinti », in English

Historical Review, 2008, CXXIII (504), 1109-1131 ; James Hamilton Wylie, The Reign of Henry the Fifth, III, Cambridge, 1929, p. 336 et suiv. La question de l'identité de l'auteur de la Vita et gesta Henrici Quinti (par Thomas d'Elmham ou un pseudo Thomas d'Elmham) de même que le rapport de cette biographie avec la Vita Henrici Quinti de Titus Livius Forojuliensis sont débattues. Il semble que l'oeuvre du pseudo Thomas soit antérieure à celle de l'Italien.

${ }^{39}$ T. Rymer, Foedera, conventiones, literae et cuiscumque generis acta publica inter reges Angliae et alios, London, 1727, X, pp. 212-214.

${ }^{40}$ B. Schnerb, Les Armagnacs, pp. 221-226.

${ }^{41}$ Un procès au Parlement ayant pour toile de fond le siège de Meaux va dans ce sens : il évoque la dureté des capitaines armagnacs envers leurs prisonniers, mais incrimine Jean d'Aunay - à qui a Henri V laisse la vie sauve - bien plus que le bâtard de Vaurus (BnF, X1a 4795, fol. 244).

${ }^{42}$ Par ailleurs, l'auteur considère l'impôt municipal de 1424 comme une « rançon » qu'il met donc au même niveau que les pillages des routiers, ce qui prouve bien que ses catégories de lecture des événements ne sont pas les mêmes que celles des historiens (Journal, § 414, 806, 842)

${ }^{43}$ M. Bloch, «Réflexion sur les fausses nouvelles de la guerre (1921), in L'histoire, la guerre, la résistance, Paris, 2006, p. 312.

${ }^{44}$ C. Gauvard, Violence, 2005, p. 246.

45 Ce dernier le fait pendre sans procès et sans confession, malgré la résistance du bourreau et l'avertissement de la Providence qui fait casser la corde lors de la première tentative. Pour justifier cette 
iniquité, le Bourgeois se borne à rapporter ce qu'en dit la propagande anglaise («on disait qu'il avait tué de sa main au pays de Flandre ou de Hainaut un évêque ») alors qu'il détaille la cruauté de l'exécution ordonnée par Bedford (Journal, § 476). On a donc le même schéma que pour Vaurus - l'exécution illégale d'un homme d'arme justifiée par ses énormes crimes - sans que l'auteur invente plus que ce qu'il sait.

${ }^{46}$ C. Brémond, J. Le Goff, J.C. Schmitt, L'Exemplum, Turnhout, 1982, pp. 37-38.

47 Journal, § 276

${ }^{48}$ C'est la règle dans les sièges médiévaux (J. Bradbury, The medieval siege, London, 1992, p. 312).

${ }^{49}$ Nicolas de Fauquembergue signale que le 26 mai, le Parlement a rejeté l'appel de la condamnation à mort du bailli de Meaux et de son lieutenant, prononcée par le prévôt de Paris (annexe).

${ }^{50}$ P. Contamine, Guerre, Etat et société à la fin du Moyen Âge, 1972, rééd. Paris, 2004, p. 262.

${ }^{51}$ Le témoignage des chroniqueurs, 20.000 hommes, doit être ici revu à la baisse, mais cela paraît de bon sens. A. H. Burne, The Agincourt War, 1956, rééd. London, 1999, p. 171.

52 I. Taddei, «Les rituels de dérision entre les villes toscanes (XIII ${ }^{\mathrm{e}}-\mathrm{XIV}^{\mathrm{e}}$ siècles) » et R. Villard, « La queue de l'âne : dérision du politique et violence en Italie dans la seconde moitié du $\mathrm{XV}^{\mathrm{e}}$ siècle » in $\mathrm{E}$. Crouzet-Pavan (dir.), La dérision au Moyen Âge, Paris, 2007, pp. 175-190 et 205-224.

${ }^{53}$ J. Bradbury, The medieval siege, 1992, p. 307.

${ }^{54}$ Référence en annexe.

${ }^{55}$ C. Gauvard, «De grace especial », p. 197, 211-213.

${ }^{56} \mathrm{Cf}$ la grande ordonnance de réforme du 3 mars 1357 (D. F. Secousse, Ordonnances, III, p. 121-146). Etienne Marcel dénonce dans les mêmes termes les crimes commis par les nobles lors de la contre Jacquerie dans sa lettre aux échevins d'Ypres du 11 juillet 1358 (Kervyn de Lettenhove, Euvres de Froissart. Chroniques, Bruxelles, 1868, VI, p. 466-472).

${ }^{57}$ C. Gauvard, «Résistants et collaborateurs pendant la guerre de Cent ans : le témoignage des lettres de rémission », in La France anglaise au Moyen Âge, Paris, 1988, p. 123-138. Ead., "De grace especial », p. $705-752$.

${ }^{58}$ V. Toureille, Vol et brigandage au Moyen Âge, Paris, 2006, p. 279-283.

${ }^{59}$ Chronique de Jean Le Bel, éd. J. Viard et E. Desprez, SHF, Paris, 1905, II, p. 255-260. Chronique de Jean Froissart, éd. S. Luce, SHF, 1874, V, p. 99-102.

${ }^{60}$ Il est révélateur de la partialité des chroniqueurs que le Religieux de Saint-Denis ne mentionne les Tuchins, pourtant actifs dès les années 1360 en Auvergne, qu'en 1384 lorsque leur activité de résistance aux soldats pillards paraît moins légitime avec l'accalmie du conflit franco-anglais. Lorsqu'il en parle enfin, c'est pour dénoncer leurs crimes (Chronique citée en annexe, I, p. 309-311). Les historiens ont un jugement plus nuancé sur ce mouvement : P. Charbonnier, "Qui furent les Tuchins ? », in Violence et contestation au Moyen Âge, Paris, 1990, p. 235-247 et V. Challet, « La révolte des Tuchins : banditisme social ou sociabilité villageoise ?», Médiévales, printemps 1998, n³4, pp. 101-112. 